\title{
Modulation instability gain and Modulated wave shape incited by the acoustic longitudinal vibrations in Molecular Chain Model
}

Alphonse Houwe ( $\square$ ahouw220@yahoo.fr)

Limbe Nautical Arts and Fisheries Institute

Philippe Djorwe

Univesrity of Ngaoundere

Youssoufa Saliou

University of Maroua

Souleymanou Abbagari

Serge Yamigno Doka

University of Ngaoundere

\section{Research Article}

Keywords:

Posted Date: February 11th, 2022

DOI: https://doi.org/10.21203/rs.3.rs-1346818/v1

License: (c) (1) This work is licensed under a Creative Commons Attribution 4.0 International License.

Read Full License 


\title{
Modulation instability gain and Modulated wave shape incited by the acoustic longitudinal vibrations in Molecular Chain Model
}

\author{
Alphonse Houwe ${ }^{a, 4 *}$, Philippe Djorwe ${ }^{c}$, Youssoufa Saliou ${ }^{b}$, Souleymanou Abbagari ${ }^{d, e}$, Serge Yamigno \\ Doka $^{c}$ \\ ${ }^{a}$ Department of Marine Engineering, Limbe Nautical Arts and Fisheries Institute, P.O Box 485, Limbe, Cameroon. \\ ${ }^{b}$ Department of Physics, Faculty of Science, The University of Maroua, P.O Box 814, Maroua, Cameroon. \\ ${ }^{c}$ Department of Physics, Faculty of Science, The University of Ngaoundere, P.O Box 454, Maroua, Cameroon. \\ ${ }^{d}$ Department of Basic Science, Faculty of Mines and Petroleum Industries, The University of Maroua, P.O Box 08, \\ kaélé, Cameroon. \\ ${ }^{e}$ Laboratory of Mechanics, Materials and Structures, Department of physics, Faculty of Science, The University of \\ Yaounde I, P.O Box 812, Yaoundé, Cameroon.
}

\begin{abstract}
The propagation of the modulated wave pattern and its instability in nonlinear medium is an important study from where experimental results are obtained. In this work we use the Nonlinear Schrödinger Equation (NLSE) with saturation nonlinearity which describes the solitonic waves in molecular chain model. We set our study on the effectiveness of the acoustic longitudinal velocity which is related to the acoustic longitudinal vibration to investigate the behavior of the modulated wave patterns as well as the modulation instability (MI) gain. Its results that the acoustic longitudinal velocity creates instability zones, increases MI bands and shortens the transition regime of the modulated wave pattern. For long time of simulation and great enough value of the acoustic longitudinal velocity the modulated waves feature chaos-like motion. Alongside of these results the acoustic longitudinal velocity obvious itself as tool control of modulated wave as well as an energy source for the propagation of diverse modulated wave shaped in molecular chain.
\end{abstract}

\section{Introduction}

Nowadays investigations of modulated wave in diverse nonlinear media have been growing in nonlinear fibers, ion-acoustic, plasma, alpha-protein and so on. [1 14. It has been more emphasized on solitary wave which is known like soliton. Soliton is wave that can propagate in long distance without any twist and can preserve its shape despite any confrontation with another. More precisely soliton is usually resulting from the confrontation between nonlinear and dispersions term of the media. However, its propagation is described

\footnotetext{
* Corresponding authors:

E-mail address:

ahouw220@yahoo.fr (A. Houwe).

djorwepp@gmail.com (P. Djorwe).

youssoufas@yahoo.fr (Y. Saliou).

abbagaris@yahoo.fr (S. Abbagari).

numami@gmail.com (S. Yamigno Doka).
} 
by the nonlinear evolution equation such as NLSE, nonlinear modified Zakharov-Kuznetsov equation of ionacoustic waves, the dissipative Kuramoto-Sivashinsky equation, the perturbed Chen-Lee-Liu equation jus to name few [1,2,4-10]. Owing the nonlinear term of the NLSE, diverse form are known in literature such as kerr law, Cubic-Quintic Nonlinearity (CQN), parabolic law including saturable nonlinearity. Nonlinear terms are thus involved in the structuring of the modulated wave patterns and act with dispersions coefficients for a perfect accomplishment of the propagation of solitonic waves (SWs). For example, it has been emphasized in molecular chain, the propagation of the bell soliton and kinks-like soliton where saturable nonlinearity (SN) is used [15, 16]. Beside, it has been shown that dromion-like soliton can be obtained in alpha-protein array when CQN act together with the acoustic longitudinal velocity (ALV). On this way, in molecular chain array diverse other studies have been successfully done by implying the SN. In refs. [17, 18, it has been shown how the saturation terms can deeply affect the modulated wave and modulation bands in a bi-exciton alpha-helical protein chains.

The nonlinear effects in molecular chain have been proposed in literature, but few of them have included the effects of the acoustic sound waves. Since the works of [16], where ALV has been used to control solitons velocity in the presence of CQN, we have set our interest on both SN and ALV to study the modulated solitary waves and MI growth rate in molecular chain. The goal of this work is to point out the effectiveness of ALV on both MI growth rate and modulated wave patterns in molecular chain with SN. The paper is organized as follows: in sect.1, we overview the model of NLSE with SN which describes the propagation of SWs in molecular chain. Sect. 2 is focussed to point out the effects of the ALV on MI bands and MI growth rate. The paper is organized as follows: in sect.1 we overview the model of NLSE with SN which describes the propagation of SWs in molecular chain. Sect. 2 is focussed to point out the effects of the ALV on MI bands and MI growth rate. Using numerical simulation, we have shown out the modulated wave pattern by varying the ALV and fixed value of the SN term during long time of simulation. Some relevant results have been obtained to confirm the effectiveness of the ALV. We give the summary in last section.

\section{Nonlinear Schrödinger equation with saturable nonlinearity}

Recently it has been derived NLSE with SN which describes the propagation of the solitonics structures in Molecular chain by the authors [15] 17]. These relevant works are based on the pipe given by the Davydov's model where interactions of neighbor are included [17]. However, to achieve the goal of this work, we consider here one-dimensional chain where interactions are considered between neighbor molecules. Following the processus uses in ref. [17] and after some calculation in continuum limit the CQN with SN gives

$$
i \hbar \frac{\partial b}{\partial t}+J \frac{\partial^{2} b}{\partial \xi^{2}}-\lambda b+\frac{k_{1}|b|^{2} b}{G-k_{3}|b|^{2}}+\frac{k_{2}|b|^{4} b}{\left(G-k_{3}|b|^{2}\right)^{2}}=0 .
$$

So, $\hbar$ is a shorten Planck's constant and $k_{1}, k_{2}$ and $k_{3}$ are respectively the attractive and repulsive nonlinearity terms and the NS term. They are connected to the exciton-phonon coupling coefficient as $k_{1} \equiv \frac{\beta^{2} D^{2}}{R^{2} M}$, $k_{2} \equiv \gamma \frac{\beta^{3} D^{3}}{2 R^{4} M^{2}}$ and $k_{3} \equiv \frac{\beta \gamma D}{R^{2} M}$. The coefficient $\lambda=\varepsilon+T+U-D-J$, while $G=v_{a}^{2}-v^{2}$. Here $v_{a}$ represents the ALV or longitudinal vibrations of molecules and $v$ the soliton velocity. The dipole-dipole interaction is given by $J=\frac{2 d^{2}}{R^{3}} . M$ represents the mass, $T$ and $U$ are respectively the kinetic and potential energies of the 
longitudinal movement. $R$ denotes the intramolecular distances and the exciton-phonon coupling is given by $\chi=\frac{\beta D}{R}$. The details of these parameters are given in [17].

More recently, Muniyappan and co-workers [16] have displayed the effects of the ALV on dromion structures in alpha-helica proteins by using the CQNLSE without SN. They have pointed out the robustness of the obtained analytical results. Beside, M. Aguero et al. [15], have established the effectiveness of different excitations in one-dimensionless array model of molecular chain. They have exhibited that the soliton velocity can be controlled by ALV which is known as longitudinal vibrations of molecules. We aim in what will be follows to examine the effects of the acoustic longitudinal vibrations on modulated wave patterns.

In the upcoming section we point up the efficiency of the ALV on both MI band and MI growth rate by using the linearizing scheme.

\subsection{Linear stability}

We assume the plane wave solution of Eq.(1) in the form

$$
b(x, t)=u_{0} e^{\imath\left(k_{0} \xi-\omega_{0} t\right)},
$$

where $u_{0}, k_{0}$ and $\omega_{0}$ are respectively the complex amplitude, the wave vector and angular frequency. Inserting Eq.(2) into Eq.(1) gives the dispersion relation as

$$
\omega_{0}=\frac{J k_{0}^{2}}{\hbar}+\frac{\lambda}{\hbar}-\frac{k_{1}\left|u_{0}\right|^{2}}{\hbar\left(G-k_{3}\left|u_{0}\right|^{2}\right)}-\frac{k_{2}\left|u_{0}\right|^{4}}{\hbar\left(G-k_{3}\left|u_{0}\right|^{2}\right)^{2}} .
$$

The obtained dispersion relation depends on the wave vector, attractive and repulsive terms. To linearize Eq.(1), we assume its solution in the form of the continuous wave (CWs) given by

$$
b(x, t)=u_{0}(1+\psi(\xi, t)) e^{\imath\left(k_{0} \xi-\omega_{0} t\right)} .
$$

Inserting Eq.(4) into Eq.(1), we get the linearizing expression as follows

$$
i \hbar \frac{\partial \psi(\xi, t)}{\partial t}+J \frac{\partial^{2} \psi(\xi, t)}{\partial \xi^{2}}+2 i k_{0} \frac{\partial \psi(\xi, t)}{\partial t}+\frac{k_{1} G\left|u_{0}\right|^{2}\left(\psi(\xi, t)+\psi^{*}(\xi, t)\right)}{\left(G-k_{3}\left|u_{0}\right|^{2}\right)^{2}}+\frac{2 k_{2} G\left|u_{0}\right|^{4}\left(\psi(\xi, t)+\psi^{*}(\xi, t)\right)}{\left(G-k_{3}\left|u_{0}\right|^{2}\right)^{3}}=0 .
$$

We aim in the forthcoming section to inspect the effects of the $v_{a}$ (acoustic longitudinal velocity) on the such MI growth rate and MI bands.

\subsection{Modulation Instability}

The Modulation instability is the event from which nonlinear and dispersion terms are combined during soliton formation in sundry media such as nonlinear optical fibers, plasma, ion-acoustic just to mention these [2-14]. Let's set the following with perturbed terms as solution of the given Eq.(5) above

$$
A=\delta_{1} e^{i(K \xi-\Omega t)}+\delta_{2} e^{-i(K \xi-\Omega t)}
$$


Here $\delta_{j}(j=1,2), K$ and $\Omega$ are respectively the complex amplitude, perturbed wave number and angular frequency. Using Eq.(6) into Eq.(5) we get the $2 \times 2$ matrix from where the dispersion relation can be obtain

$$
\left(\begin{array}{cc}
\Omega \hbar-J K^{2}-2 k_{0} K+M & M \\
M & -\Omega \hbar-J K^{2}+2 k_{0} K+M
\end{array}\right)\left(\begin{array}{l}
\delta_{1} \\
\delta_{2}
\end{array}\right)=\left(\begin{array}{l}
0 \\
0
\end{array}\right)
$$

with

$$
M=\frac{k_{1}\left|u_{0}\right|^{2}}{\left(G-k_{3}\left|u_{0}\right|^{2}\right)^{2}}+\frac{k_{2}\left|u_{0}\right|^{4}}{\left(G-k_{3}\left|u_{0}\right|^{2}\right)^{3}} .
$$

The obtained matrix vanishes when the following constraint relation is satisfied

$$
\lambda_{2} \Omega^{2}+\lambda_{1} \Omega+\lambda_{0}=0
$$

and

$$
\begin{aligned}
& \lambda_{2}=\frac{\left(\left|u_{0}\right|\right)^{6} \hbar^{2} k_{3}{ }^{3}-3 G\left(\left|u_{0}\right|\right)^{4} \hbar^{2} k_{3}^{2}+3 G^{2}\left(\left|u_{0}\right|\right)^{2} \hbar^{2} k_{3}-G^{3} \hbar^{2}}{\left(-\left(\left|u_{0}\right|\right)^{2} k_{3}+G\right)^{3}}, \\
& \lambda_{1}=\frac{-4 K\left(\left|u_{0}\right|\right)^{6} \hbar k_{0} k_{3}^{3}+12 G K\left(\left|u_{0}\right|\right)^{4} \hbar k_{0} k_{3}^{2}-12 G^{2} K\left(\left|u_{0}\right|\right)^{2} \hbar k_{0} k_{3}+4 G^{3} K \hbar k_{0}}{\left(G-\left|u_{0}\right|^{2} k_{3}\right)^{3}}, \\
& \lambda_{0}=\frac{-J^{2} K^{4}\left(\left|u_{0}\right|\right)^{6} k_{3}^{3}+3 G J^{2} K^{4}\left(\left|u_{0}\right|\right)^{4} k_{3}^{2}+4 K^{2}\left(\left|u_{0}\right|\right)^{6} k_{0}^{2} k_{3}^{3}-3 G^{2} J^{2} K^{4}\left(\left|u_{0}\right|\right)^{2} k_{3}-12 G K^{2}\left(\left|u_{0}\right|\right)^{4} k_{0}{ }^{2} k_{3}^{2}}{\left(G-\left|u_{0}\right|^{2} k_{3}\right)^{3}} \\
& +\frac{2 G J K^{2}\left(\left|u_{0}\right|\right)^{4} k_{1} k_{3}+G^{3} J^{2} K^{4}+12 G^{2} K^{2}\left(\left|u_{0}\right|\right)^{2} k_{0}{ }^{2} k_{3}-4 G J K^{2}\left(\left|u_{0}\right|\right)^{4} k_{2}-2 G^{2} J K^{2}\left(\left|u_{0}\right|\right)^{2} k_{1}-4 G^{3} K^{2} k_{0}^{2}}{\left(G-\left|u_{0}\right|^{2} k_{3}\right)^{3}} .
\end{aligned}
$$

In figure 1a, b, c and d we display the behavior of the MI gain with the effects of the acoustic longitudinal velocity and fixed value of soliton velocity. In figure $1 \mathrm{a}-\mathrm{b}$, we assume $v_{a}>v$ (velocity of the bright soliton) and fixed values of attractive and repulsive nonlinearity. We have shown how the MI band increases both in amplitude and wave number range, for $v_{a}=4.8[\hbar]$ and $v_{a}=6.8[\hbar]$ respectively. Alongside of figure 1a-b, we have used the reverse situation in figure $1 \mathrm{c}$-d (i.e $v_{a}<v$ ). It is observed decreasing MI growth rate both in amplitude and wave number range compared to figure 1a-b. More precisely for $v=10.3[\hbar]$ and fixed value of $v_{a}=4.8[\hbar]$ we have observed in figure1c the MI growth rate with decreasing amplitude and wave number range between $[0,1]$. Further, we have increased the soliton velocity to $v=20.3[\hbar]$ and fixed value of the acoustic longitudinal velocity, we have pointed that the MI growth rate decreases and vanishes for $v=25.3[\hbar]$. It could be predicted that the ALV can provoke unstable continuous wave during the formation of modulated waves in molecular chain. However, the situation could be supposedly controlled by increasing the soliton velocity. In the next section, after some assumption, we use the transformation hypothesis to obtain bright soliton as initial condition for numerical simulation. At the end we examine numerical its behavior like modulated wave pattern under the effects of the longitudinal acoustic vibration. 

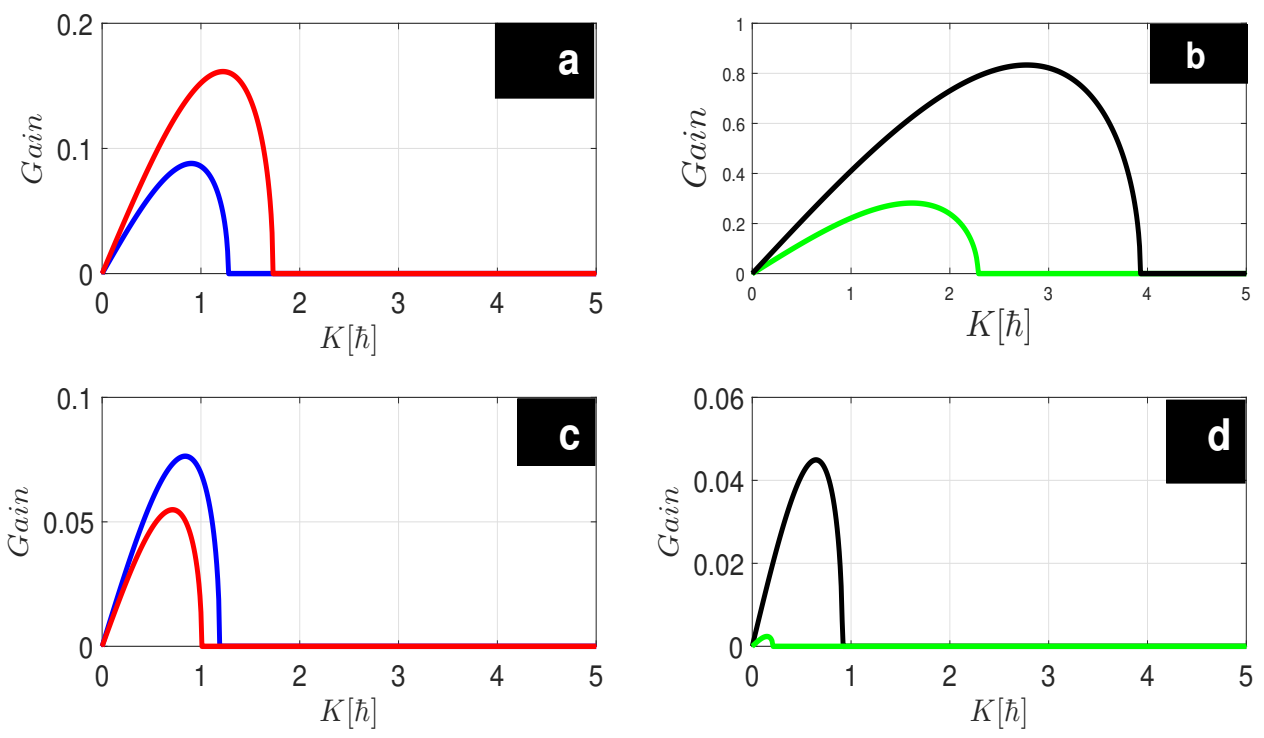

Figure 1: Effects of the ALV $\left(v_{a}\right)$ on MI bands. (a-b) are variation of the velocity sound while (c-d) denotes the variation of the soliton velocity. The parameters use are $J=1.08[\hbar], k_{2}=1.25[\hbar], k_{3}=1.3[\hbar], k_{0}=1.5$.

\subsection{Numerical analysis of modulated wave pattern}

. In what follows we gaol to examine the effectiveness of the numerical simulation on modulated wave pattern propagating in nonlinear saturation alpha-helical proteins. It is worth to highlight that Eq.(1) can obey to some analytical solutions where the nonlinearity is less than $O\left(k_{3}|b|^{2}\right)$ and $k_{3} \ll k_{1} k_{2}$. To achieve this assumption, we apply the transformation in the form of

$$
b(x, t)=f(x-v t) e^{\imath(\kappa x+\omega t)},
$$

where $\zeta=x-v t$. The parameters $f(x-v t), \kappa$ and $\omega$ are respectively the complex amplitude of the modulated wave, the vector wave and the angular frequency respectively. Owing to the assumption of $k_{3} \ll k_{1} k_{2}$, we insert Eq.(11) into $\mathrm{Eq}(1)$ the soliton velocity is obtained

$$
v=\frac{2 J \kappa}{\hbar} \text {. }
$$

Subsequently the ordinary equation relate to Eq.(1) is set in the form of

$$
f^{\prime \prime}(\zeta)+\alpha_{0} f(\zeta)+\alpha_{1} f^{\sigma+1}(\zeta)+\alpha_{2} f^{2 \sigma+1}(\zeta)=0,
$$

where $\sigma=2$, along with the constant coefficients

$$
\begin{aligned}
& \alpha_{0}=-\frac{J \kappa^{2}-\hbar \omega+\lambda}{J}, \\
& \alpha_{1}=\frac{k_{1}}{G J}, \\
& \alpha_{2}=\frac{k_{2}}{J G^{2}} .
\end{aligned}
$$

Following refs. [19,20], Eq.(13) can satisfy bright-like soliton and a pair of kink-profile as solutions by using the undermined coefficients method. It has been also pointed out four exact solutions of Eq.(13) in 
ref. [20]. We aim here to show only the effects of the ALV on modulated wave patterns, we only consider one of the solution of Eq.(13). For this fact, we have $\sigma=2$ and then we assume the following constraint relation

$$
\begin{aligned}
& \alpha_{0}<0, \\
& \alpha_{1}=0, \\
& \alpha_{2}>0 .
\end{aligned}
$$

Hence the following solution is obtained

$$
b(x, t)=\sqrt{\sqrt{\frac{3\left(\frac{J \kappa^{2}-\hbar \omega+\lambda}{J}\right)}{\frac{k_{2}}{J G^{2}}}} \operatorname{sech}\left(2 \sqrt{\frac{J \kappa^{2}-\hbar \omega+\lambda}{J}}(x-v t)\right)} e^{\imath(\kappa x+\omega t) .}
$$

It is obvious that the obtained bright soliton-like solution depends on dipole-dipole interactions coefficient, the acoustic longitudinal velocity as well as the repulsive nonlinearity and the reduced Planck's constant. Let now focuss on numerical investigation of Eq.(1) with the initial condition Eq.(16) to point out the efficiency of the velocity of sound waves corresponding to longitudinal vibrations of molecules on the modulated wave patterns. We assume for this $x_{0}=n / 2$. To carry out the effects of ALV on the modulated waves patterns, we have fixed the other parameters related to the alpha-helica proteins. For this purpose we set the vector wave $\kappa=0$ and therefore the soliton velocity is $v=0$. In figure 2a-f we examine the behavior of the modulated wave under the effects of the acoustic longitudinal velocity. For $v_{a}=0.5[\hbar]$, we observe the formation of breather-like soliton with concentrate energy at $n / 2$ in figure $2 \mathrm{a}$, while the breathing behavior is pointed out in figure $2 \mathrm{~d}$ for $T \epsilon[0,500[\hbar]]$. In order to fully appreciate the behavior of the modulated wave, we increase the ALV to $v_{a}=0.6[\hbar]$, the breathing behavior have extended in time propagation in figure $2 \mathrm{e}$ and a hight concentration of energy is obtained at $n / 2$ in figure $2 \mathrm{~b}$. In addition, it also appears a less disorderly behavior for $T \rightarrow 1500[\hbar]$ in Figure 2e. Once more, we increase $v_{a}=0.7[\hbar]$, we observe nonlinear propagation of the modulated wave in figure $2 \mathrm{c}$ and for $\mathrm{n}=100$, we have pointed out in figure $1 \mathrm{f}$ the chaotic behavior in molecular chain. In figure 2, we maintain the long time of propagation and fixed value of the ALV and of course with zero soliton velocity. We portray the soliton-like modulated wave in terms of cell index. In figure 3a-c for $T=10[\hbar], T=250[\hbar]$ and $T=750[\hbar]$ the chaotic propagation of the modulated wave is fulfilled. We portray the soliton-like modulated wave in terms of cell index. On the bottom panel of figure 3 , we have shown the propagation of the modulated wave pattern such as bell-shaped in figure $3 \mathrm{~d}$ for $\mathrm{n}=120$, dipole soliton in figure $3 e$ where $n=150$ and $W$-shaped profile in figure $3 f$ for cell index $n=200$. We have observed modulated wave patterns with decreasing amplitude during the propagation in molecular chain. The decrease in amplitude observed as a function of cell index could also reflect a loss of energy during the propagation of the modulated waves in the molecular chain. To better appreciate the different shapes of the modulated wave we display the propagation of the intensity of the modulated wave at different time in figure 4a-f. We set $T=150[\hbar]$ in figure $4 \mathrm{a}$, the $\mathrm{W}$-shaped profile is obtained at the center of the cell index. We increase the propagation time to $T=450[\hbar]$ the M-shaped is observed with increasing amplitude. To better appreciate different shapes of the modulated waves patterns with time of propagation, we have increased the propagation time such as $T=550[\hbar], T=750[\hbar], T=900[\hbar]$ and $T=950[\hbar]$ respectively in figures $4 \mathrm{~d}$-f. We have shown $\mathrm{W}$-shaped with one peak of concentrate energy and with a reduced width in figure $4 \mathrm{~d}$. More particularly, for $T=950[\hbar]$, it is appeared a soliton-like rogue wave with hight amplitude 

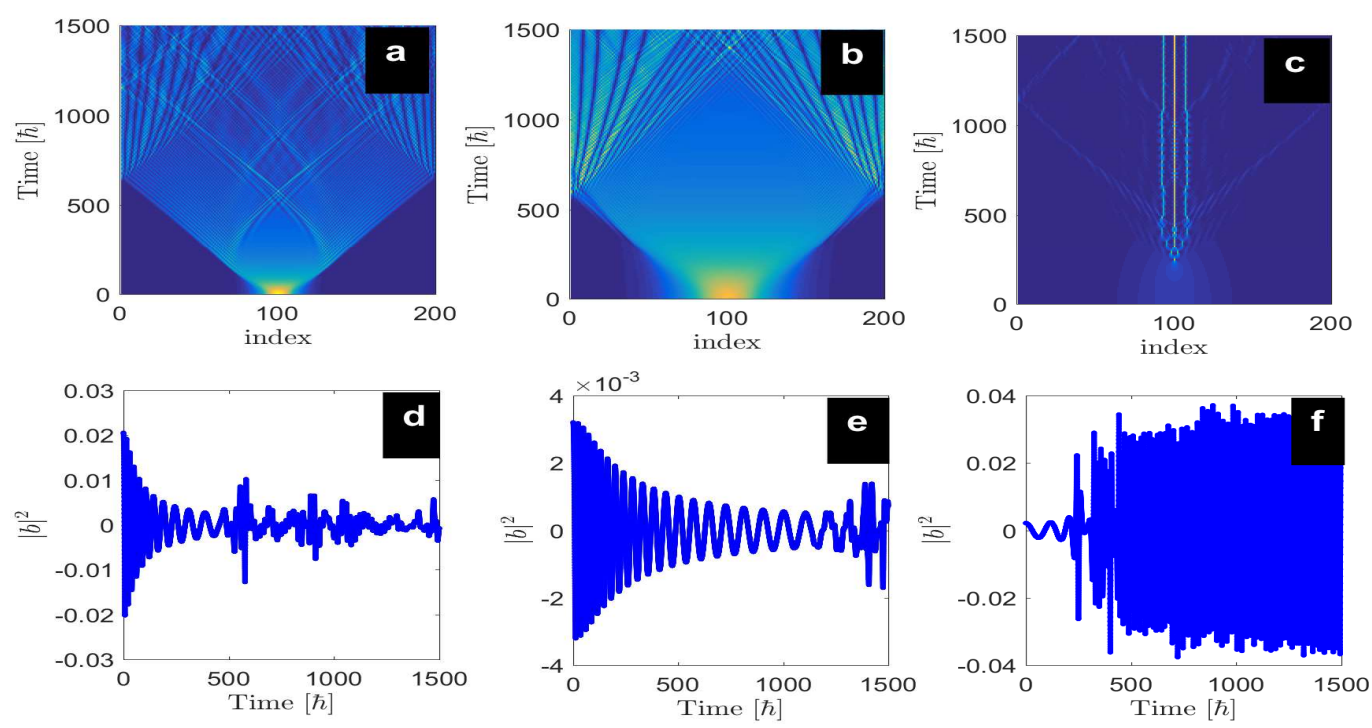

Figure 2: Effects of acoustic longitudinal velocity $\left(v_{a}\right)$ on soliton. $(\mathrm{a}, \mathrm{b}, \mathrm{c})$ are the temporal soliton in terms of the cell index and (d, e,f) are the behavior of the temporal soliton evolution in terms of time at different cell index (d, e, f). (a) $v_{a}=0.5[\hbar]$, (b) $v_{a}=0.6[\hbar]$, (c) $v_{a}=0.7[\hbar]$, (d) $n=20$, (e) $n=50$, (f) $n=100$. The other parameters are $J=0.08[\hbar], \lambda=0.1[\hbar], k_{2}=-1.2[\hbar], k_{3}=0.4[\hbar], v=0, \omega=0.8[\hbar], \kappa=0$.

and small width. After increasing deeply the propagation time to $T=950[\hbar]$, it is shown how the amplitude of the obtained new shaped in figure $4 \mathrm{f}$ increases. To highlight the vector wave effects on the modulated wave patterns we set $\kappa=\frac{\pi}{6}$ and $\kappa=-\frac{\pi}{6}$ respectively. For this event we have fixed the ALV value. From fig5a-c we set $\kappa=\frac{\pi}{6}$ and the velocity of the soliton is $v=1.13[\hbar]>v_{a}$. It is observed the propagation of the modulated forward wave in figure $5 \mathrm{a}$ while in figure $5 \mathrm{~b}$ the bright-like soliton is fulfilled. At the same time we have shown the breathing behavior of the modulated wave pattern in figure 5c. From the bottom panel figure $5 \mathrm{~d}$-f, we reverse the sign of the vector wave $\kappa=-\frac{\pi}{6}$ and the $v=-1.13[\hbar]<v_{a}$, we have shown the propagation of the modulated backward wave (i.e. group velocity and phase velocity are conversed). This is one of the promising results in molecular chain which may open up a view of the propagation of backward waves which have been demonstrated in media with a negative refractive index.

We can predict that during propagation of the modulated wave pattern the ALV has a lot of effects on the soliton-like solution and for long time of propagation in molecular chain there is energy transfer between neighbor and the modulated wave can change shape. Otherwise, despite the soliton velocity is sometime zero we have observed how the modulated wave pattern is affected in time and can also preserve hight energy.

\section{Conclusion}

In this study we have shown the effects of the acoustic longitudinal velocity on the modulation instability growth rate and modulated wave patterns in molecular chain where saturable nonlinearity is considered. We have shown on MI bands and modulated wave pattern the effectiveness of ALV. For this purpose we use 

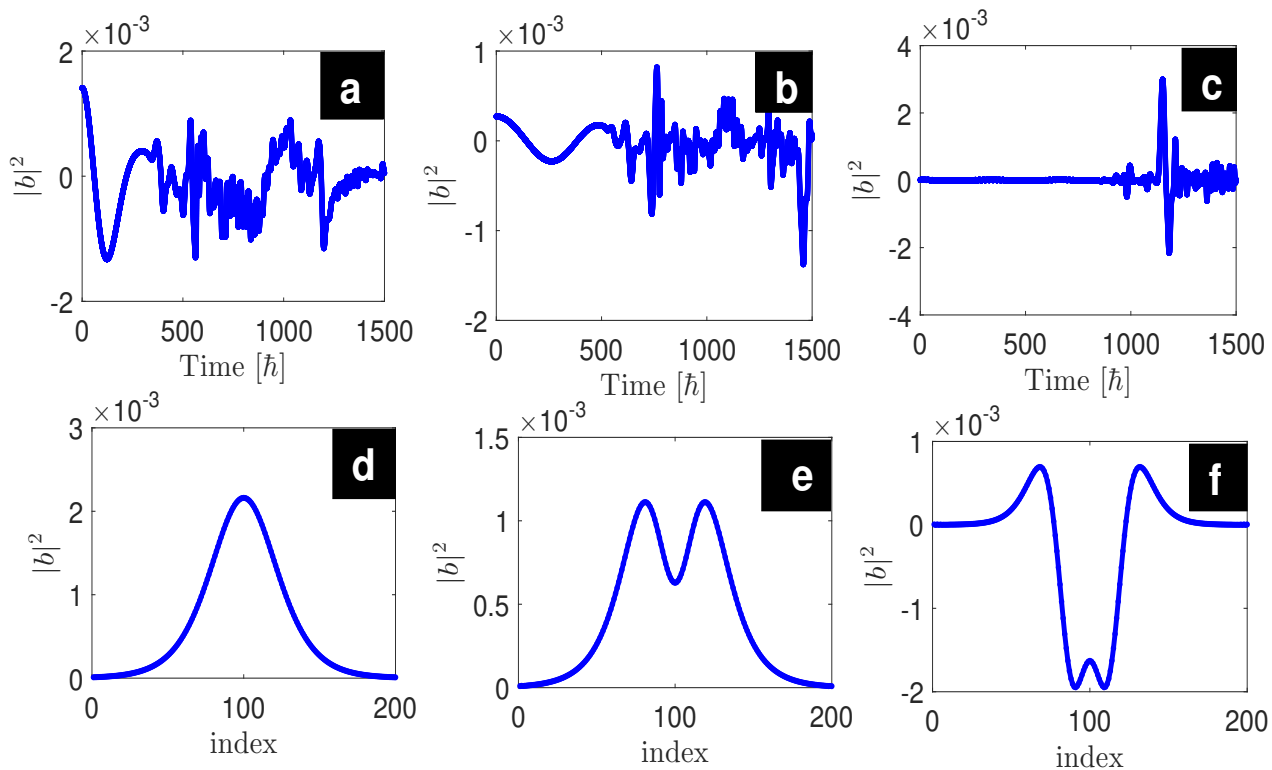

Figure 3: Soliton evolution with fixed value of acoustic longitudinal velocity. (a, b, c) are the respective values of the cell index and (d, e, f) are the respective time of propagation. (a) $T=10[\hbar]$, (b) $T=250[\hbar]$, (c) $T=750[\hbar]$, (d) $n=120$, (e) $n=150$, (f) $n=200$. The other parameters are $v_{a}=0.7[\hbar], J=0.08[\hbar], \lambda=$ $0.1, k_{2}=-1.2[\hbar], k_{3}=0.4[\hbar], v=0, \omega=0.8[\hbar], \kappa=0$.
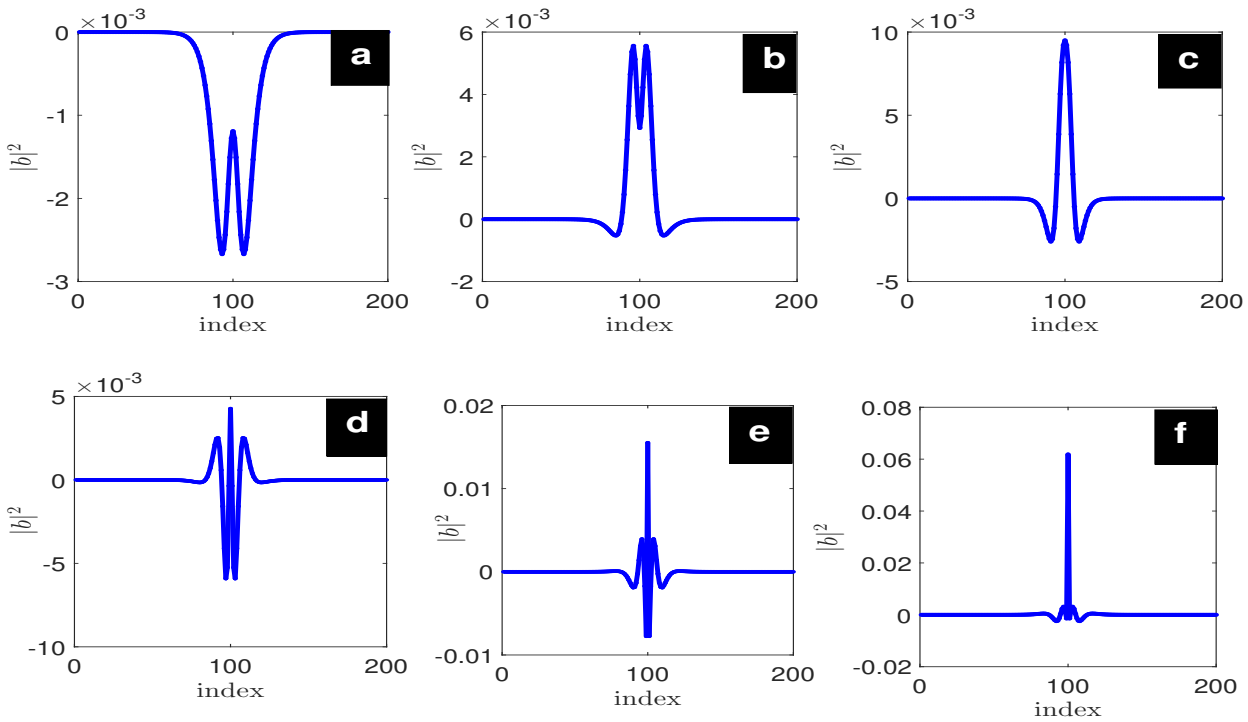

Figure 4: Dynamics of bright solitons in terms of cell index at different time of propagation with fixed value of the velocity sound waves. a, b, c, d and f correspond respectively to $T=150[\hbar], T=450[\hbar], T=550[\hbar]$, $T=750[\hbar], T=900[\hbar]$ and $T=950[\hbar]$. The other parameters are $v_{a}=0.6[\hbar], J=0.08[\hbar], \lambda=0.1, k_{2}=$ $-1.2[\hbar], k_{3}=0.4[\hbar], v=0, \omega=0.8[\hbar], \kappa=0$. 

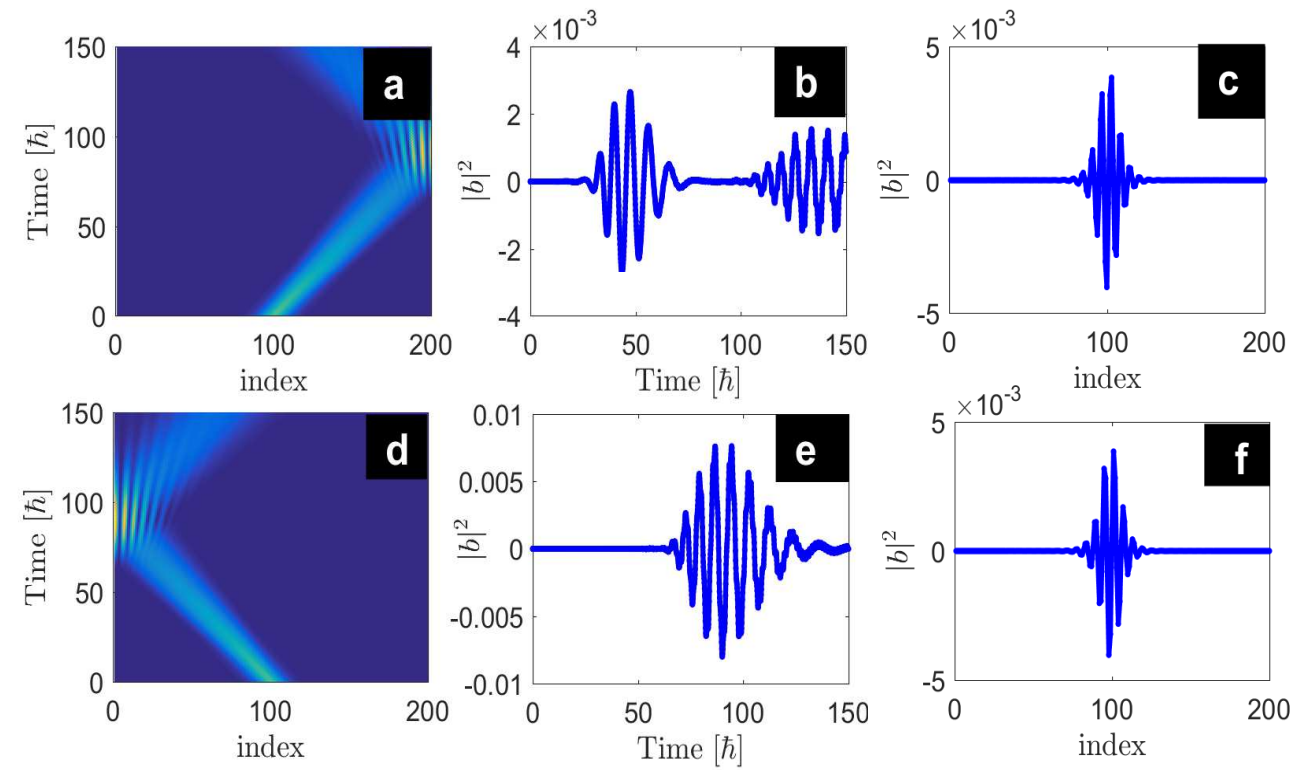

Figure 5: Soliton propagation with the variation of wave number $(\kappa)$. For $(\mathrm{a}, \mathrm{b}, \mathrm{c}) \kappa=\frac{\pi}{6}$ and $(\mathrm{d}, \mathrm{e}, \mathrm{f})$ we set $\kappa=-\frac{\pi}{6}$. (b, e) are obtained where the cell index value is respectively $n=150$ and $n=1$ while $(c, f)$ is displayed for $T=10[\hbar]$. The other parameters are $v_{a}=0.9[\hbar], J=1.08[\hbar], \lambda=0.1,, k_{2}=1.8[\hbar], k_{3}=$ $1.1[\hbar], \omega=0.8[\hbar]$.

numerical simulation to show for long time of simulation the chaos-like motion. We have established for strong value of ALV the propagation of dipole soliton, W-shaped profile as well as soliton-like rogue wave. These results have also shown the feature of backward wave for negative value of the soliton velocity and the acoustic sound waves behaves as being an energy source for the modulated wave patterns during long time of simulation. This study open the gate to the new behavior of the modulated wave like reverse wave in molecular chain.

\section{References}

[1] H. Susanto, B.A. Malomed, Embedded solitons in second-harmonic-generating lattices, Chaos, Solitons and Fractals 142 (2021) 110534.

[2] C. Bertrand Tabi, Fractional unstable patterns of energy in a-helix proteins with long-range interactions. Chaos Solitons Fractals 116 (2018) 386-391.

[3] P. K. Shukla, L. Stenflo \& R. Fedele, Nonlinear effects caused by intense electromagnetic waves in an electron-positron-ion plasma, Phys. Plasmas 10 (2003) 310.

[4] A. R. Seadawy, Three-dimensional nonlinear modified Zakharov-Kuznetsov equation of ion-acoustic waves in a magnetized plasma, Com. Math. Appl. 71 (2016) 201-212. 
[5] N. A. Kudryashov, Quasi-exact solutions of the dissipative Kuramoto-Sivashinsky equation, Appl. Math. Comput. 219 (2013) 9245-9253.

[6] A. Houwe, S. Abbagari, S. Nestor, M. Inc, Mir Sajjad Hashemi, G. Betchewe \& S. Y. Doka, Optical soliton and weierstrass elliptic function management to parabolic law nonlinear directional couplers and modulation instability spectra, Optical and Quantum Electronics 53(8)(2021) 417.

[7] D. N. Christodoulides, R. I. Joseph, Discrete self-focusing in nonlinear arrays of coupled waveguides, Opt. Lett. 13(1988) 794-796.

[8] J. H. Li, K. S. Chiang, K. W. Chow, Modulation instabilities in two-core optical fibers, J. Opt. Soc. Am. B 28 (2011) 1693-1701.

[9] A. Souleymanou, A. Houwe, D. Douvagai, S. Y. Doka \& T. C, Kofane, M-shape and W-shape bright incite by the fluctuations of the polarization in a-helix protein, Physica Scripta 96(8) (2021).

[10] A. Houwe, S. Abbagari, B. Almohsen, G. Betchewe, M. Inc \& S. Y. Doka, Chirped solitary waves of the perturbed Chen-Lee-Liu equation and modulation instability instability in optical monomode fibres. Optical and Quantum Electronics. 53(2021) 286.

[11] A. Houwe et al. Survey of third- and fourth-order dispersions including ellipticity angle in birefringent fibers on W-shaped soliton solutions and modulation instability analysis, European Physical Journal Plus 136 (4) (2021) 357.

[12] S. G. Murdoch, R. Leonhardt, J. D. Harvey, Polarization modulation instability in weakly birefringent fibers, Opt. Lett. 20 (1995) 866-868.

[13] A. Souleymanou, A. Houwe, S. P. Takougoum Mukam, H. Rezazadeh, M. Inc, S. Y. Doka \& T. B. Bouetou, Optical solitons to the nonlinear Schrödinger equation in metamaterials and modulation instability, European Physical Journal Plus 136(7)(2021) 710.

[14] A. Souleymanou, A. Houwe, D. Douvagai, S. Y. Doka \& T. C. Kofane, M-shape and W-shape bright incite by the fluctuations of the polarization in a-helix protein, Physica Scripta 96(8) (2021).

[15] M. Aguero, 'R. García-Salcedo, J. Socorro, E. Villagran, Soliton Structures in a Molecular Chain Model with Saturation, Int J Theor Phys 48 (2009) 670-683, DOI 10.1007/s10773-008-9842-7.

[16] A. Muniyappan, A. Suruthi, B. Monisha, N. Sharon Leela, J. Vijaycharles, Dromion-like structures in a cubic-quintic nonlinear Schrödinger equation using analytical methods, Nonlinear Dyn 104 (2021) 1533-1544, https://doi.org/10.1007/s11071-021-06350-1.

[17] L. Q. Kong, J. Liu, D. Q. Jin, D. J. Ding, C. Q. Dai, Soliton dynamics in the three-spine a-helical protein with inhomogeneous effect. Nonlinear Dyn. 87 (2017) 83-92.

[18] E. Simo, T.C. Kofane, Nonlinear effects in molecular chains with two types of intramolecular vibrations. Phys.Rev.E 54 (1996) 207. 
[19] E. Magyari, Kinks and Periodons at a $T=0$ First-Order Phase Transition Point in One-dimensional Anharmonic Lattices. Z. Phys. B-Condensed Matter 43 (1981) 354-351.

[20] A. Houwe, S. Abbagari, K. S. Nisar, M. Inc \& S. Y. Doka, Influence of fractional time order on Wshaped and Modulation Instability gain in fractional Nonlinear Schrödinger Equation, Results in Physics (2021) 104556.

[21] J.-H. Gan, H. Xiong, L.-G. Si, X.-Y. Lü \& Y. Wu, Solitons in optomechanical arrays, Optics Letters 41 20162676 . 